\title{
Prevalência de hiperglicemia em idosos: um estudo de base populacional
}

\author{
Prevalence of hyperglycemia in the elderly: a population-based study
}

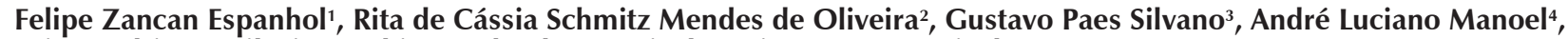 \\ Laise Rodrigues Silveira $^{3}$, Fabiana Schuelter-Trevisol ${ }^{5}$, Daisson José Trevisol ${ }^{4} \bowtie$ \\ ${ }^{1}$ Secretaria Municipal da Saúde e Assistência Social de Palmitinho. Palmitinho, RS. \\ 2 Fundação Municipal de Saúde, Prefeitura Municipal de Tubarão. Tubarão, SC. \\ ${ }^{3}$ Curso de Medicina da Universidade do Sul de Santa Catarina. Tubarão, SC. \\ ${ }^{4}$ Centro de Pesquisas Clínicas do Hospital Nossa Senhora da Conceição. Tubarão, SC \\ ${ }_{5}^{5}$ Programa de Pós-Graduação em Ciências da Saúde da Universidade do Sul de Santa Catarina. Tubarão, SC.
}

\section{RESUMO}

Objetivos: Avaliar a prevalência de hiperglicemia e fatores associados, na população idosa residente de um município do sul do Brasil.

Métodos: Estudo transversal, de base populacional, realizado com idosos ( $\geq 60$ anos) residentes na cidade de Tubarão, estado de Santa Catarina, no período de setembro de 2010 a maio de 2011. Os idosos foram cadastrados pelos Agentes Comunitários de Saúde do Programa Estratégia Saúde da Família. Foram registrados dados sociodemográficos (idade, gênero, cor da pele, situação conjugal, situação de trabalho e escolaridade), dados comportamentais (atividade física, uso de álcool e tabagismo) e dados clínicos (obesidade, uso de medicamentos e história familiar de diabetes). Após responderem estas questões, os participantes eram agendados para comparecerem à unidade de saúde para coleta de sangue e medidas antropométricas. A prevalência de hiperglicemia foi avaliada a partir de exames de glicemia de jejum e foram considerados hiperglicêmicos os indivíduos com valores $\geq 126 \mathrm{mg} / \mathrm{dL}$ ou em uso de medicamentos hipoglicemiantes. Os participantes foram selecionados por amostragem aleatória simples. Para verificar associação entre as variáveis de interesse, foi aplicado o teste do qui-quadrado. O intervalo de confiança pré-estabelecido foi de $95 \%$ e o erro $\alpha$ de $5 \%$.

Resultados: Foram incluídos 833 idosos, e 220 foram considerados hiperglicêmicos conforme os critérios da metodologia (prevalência de 26,4\%). Dos pacientes inclusos no grupo hiperglicemia, 190 estavam em uso de hipoglicemiantes orais, e destes, 112 ainda apresentavam glicemia de jejum $\geq 126 \mathrm{mg} / \mathrm{dL}$, enquanto 78 apresentavam glicemia de jejum $<126 \mathrm{mg} / \mathrm{dL}$. Ainda no grupo hiperglicemia, 30 pacientes apresentavam glicemia de jejum $\geq 126 \mathrm{mg} / \mathrm{dL}$ e não estavam em uso de hipoglicemiantes. Possuir pais com diagnóstico prévio de diabetes mellitus e ser obeso foram fatores associados à presença de hiperglicemia.

Conclusões: Este estudo sugere que uma grande parcela da população idosa tem hiperglicemia de jejum, necessitando de uma avaliação mais ampla para pesquisa do diabetes mellitus.

DESCRITORES: idoso; hiperglicemia; diabetes mellitus.

\section{ABSTRACT}

Aims: To evaluate the prevalence of hyperglycemia and associated factors in the elderly population of a municipality in southern Brazil. Methods: Cross-sectional population-based study, carried out with the elderly ( $\geq 60$ years) living in the city of Tubarão, state of Santa Catarina, Brazil, from September 2010 to May 2011. Participants were selected by simple random sampling. The elderly were registered by the Community Health Agents of the Family's Health Strategy Program. Sociodemographic data (age, gender, skin color, marital status, employment status and education), behavioral data (physical activity, alcohol use and smoking) and clinical data (obesity, drug use and family history of diabetes) were recorded. After answering these questions the participants were scheduled to attend the clinic for blood collection and anthropometric measurements. The prevalence of hyperglycemia was assessed from fasting blood glucose tests, and individuals with values $\geq 126 \mathrm{mg} / \mathrm{dL}$ or use of hypoglycemic drugs were considered hyperglycemic. To assess the association between the variables of interest, the chi-square test was applied. The pre-determined confidence interval was of $95 \%$ and the error $\alpha$ of $5 \%$.

Results: Eight hundred thirty-three elderly patients were included, and 220 were considered hyperglycemic according to the methodology criteria (prevalence of 26.4\%). Of the patients included in the hyperglycemia group, 190 were using oral hypoglycemic drugs, and of these, 112 had also fasting glucose $\geq 126 \mathrm{mg} / \mathrm{dL}$, while in 78 fasting glucose was $<126 \mathrm{mg} / \mathrm{dL}$. Also in the hyperglycemia group, 30 patients had fasting glucose $\geq 126 \mathrm{mg} / \mathrm{dL}$ and were not on hypoglycemic treatment. Having parents diagnosed with diabetes and being obese were associated with the presence of hyperglycemia.

Conclusions: This study suggests that a large portion of the elderly population has fasting hyperglycemia, requiring a wider evaluation for diabetes mellitus.

KEY WORDS: elderly; hyperglycemia; diabetes mellitus. 
Abreviaturas: DM, diabetes mellitus; DM2, diabetes mellitus tipo 2; ESITU, Estudo de Saúde dos Idosos de Tubarão.

\section{INTRODUÇÃO}

O diabetes mellitus (DM) é um grupo heterogêneo de distúrbios metabólicos caracterizados por hiperglicemia, resultante de defeitos na ação da insulina, na secreção de insulina, ou em ambas. Atualmente, o DM é categorizado em quatro classes clínicas: DM tipo 1, DM tipo 2 (DM2), DM gestacional e outros tipos específicos de DM. Essa classificação foi proposta pela Organização Mundial da Saúde e pela Associação Americana de Diabetes e é recomendada pela Sociedade Brasileira de Diabetes [1-3].

O DM é destaque na agenda global de saúde. Atualmente, estima-se que 415 milhões de pessoas convivam com essa condição, o que corresponde a $8,8 \%$ da população adulta. A maioria desses pacientes possui DM2, uma condição passível de prevenção. Foram gastos pelo menos 673 bilhões de dólares globalmente em 2015 com o tratamento e manejo das complicações do DM. No entanto, somente 19,0\% desse valor foi investido por países de baixa e média renda, que englobam $75,4 \%$ da população com DM [4].

O DM é responsável por uma redução importante na qualidade de vida e representa uma das principais causas de mortalidade, amputação de membros inferiores, doença cardiovascular e insuficiência renal [5]. A principal causa de morte nesses pacientes é o infarto agudo do miocárdio [6].

Os critérios atuais da Sociedade Brasileira de Diabetes para diagnóstico de DM são: hemoglobina glicada $\geq 6,5 \%$, glicemia de jejum $\geq 126 \mathrm{mg} / \mathrm{dL}$, glicemia duas horas após $75 \mathrm{~g}$ de glicose $\geq 200 \mathrm{mg} / \mathrm{dL}$ ou glicemia casual $\geq 200 \mathrm{mg} / \mathrm{dL}$ com sintomas clássicos de DM. Na ausência de hiperglicemia inequívoca com descompensação metabólica aguda ou sintomas óbvios de DM, o diagnóstico da doença deve ser confirmado pela repetição do teste em outro dia. $\mathrm{O}$ teste em jejum é realizado após um período de no mínimo oito horas com a falta de ingestão calórica e a glicemia plasmática casual é aquela realizada a qualquer hora do dia, sem se observar o intervalo desde a última refeição [3].

O envelhecimento apresenta tendência ao aumento da gordura, em especial na região abdominal, que associada à diminuição da massa magra gera um quadro favorável ao aumento da resistência à insulina (redução da sensibilidade tecidual à ação do hormônio) com risco para ocorrência de DM e doenças cardiovasculares. A resistência insulínica também está associada com hipertensão arterial, dislipidemia, doença hepática gordurosa não alcoólica, apneia obstrutiva do sono e câncer $[7,8]$.

Embora a carga do DM seja frequentemente descrita em termos do seu impacto na população em idade ativa, o DM em idosos está ligado a maior mortalidade, redução da capacidade funcional e aumento do risco de institucionalização [9]. Além disso, a prevalência e os custos associados com o DM desafiam a integridade financeira dos sistemas de saúde. No entanto, seu rastreio permitiria adequar a gestão da saúde pública na prevenção e manuseio das complicações relacionadas com essa condição, ao proporcionar uma abordagem mais custo-efetiva e econômica [10].

Diante do exposto, o presente estudo teve por objetivo estimar a prevalência e os fatores associados à hiperglicemia na população idosa residente em um município do sul do Brasil, como um rastreamento inicial para DM nessa população.

\section{MÉTODOS}

Foi realizado um estudo epidemiológico com delineamento transversal. A amostra estudada foi proveniente do projeto ESITU (Estudo de Saúde dos Idosos de Tubarão), realizado entre setembro de 2010 e maio de 2011. O ESITU é um estudo de base populacional que teve por objetivo avaliar a situação de saúde e qualidade de vida dos idosos residentes no município de Tubarão, estado de Santa Catarina. Este tipo de estudo é inédito no município e busca elaborar ações preventivas para os problemas possivelmente encontrados.

Tubarão é um centro regional comercial e de saúde, sendo a cidade sede da Associação de Municípios da Região de Laguna (AMUREL), que abrange 18 municípios do estado de Santa Catarina [11]. De acordo com o último censo de 2010 do Instituto Brasileiro de Geografia e Estatística, existem 97.235 habitantes em Tubarão, com uma estimativa para 2016 de 103.674 pessoas. Este total está distribuído em 32.460 domicílios nos 23 bairros da cidade, agrupados em 30.197 famílias. Da população geral, 12.017 possuem 60 anos ou mais [12].

Foram incluídas no estudo pessoas com idade $\geq 60$ anos, residentes no município de Tubarão e que aceitaram participar do estudo. Foram excluídos os indivíduos impossibilitados de responder aos questionamentos feitos ou sem autonomia para decidir sobre sua participação no estudo. 
Os participantes foram selecionados por amostragem aleatória simples. Os idosos foram cadastrados pelos Agentes Comunitários de Saúde do Programa Estratégia Saúde da Família. Considerando o número total de idosos residentes no município e uma prevalência desconhecida do desfecho de $50 \%$, o tamanho mínimo calculado para este estudo foi de 373 idosos para um nível de confiança de $95 \%$.

Os 250 Agentes Comunitários de Saúde, que visitam todas as residências do referido município mensalmente, fizeram o convite aos idosos sorteados para participar do estudo mediante anuência do Termo de Consentimento Livre e Esclarecido. O guia de questões aplicado por ocasião da visita, após o consentimento do participante, continha dados sociodemográficos (idade, gênero, cor da pele, situação conjugal, situação de trabalho e escolaridade), dados comportamentais (atividade física, uso de álcool e tabagismo) e dados clínicos (obesidade, uso de medicamentos e história familiar de DM).

Foi considerado tabagista o indivíduo que referiu ter consumido 100 ou mais cigarros durante toda sua vida [13]. Nos idosos que afirmaram consumir álcool atualmente ou no passado, foi aplicado o questionário CAGE. Esse instrumento é composto de quatro perguntas, sendo duas ou mais respostas positivas um indicativo de alcoolismo [14]. Foram considerados fisicamente ativos os indivíduos que realizavam no mínimo 30 minutos de atividade física em cinco dias da semana, resultando em 150 minutos ou mais de atividade física semanal, independentemente do tipo ou intensidade [15].

Respondidas estas questões, foi agendada uma data para comparecimento da pessoa à unidade de saúde, para coleta de sangue e das medidas antropométricas. Todas as amostras foram encaminhadas para o mesmo laboratório, e para determinação da glicemia foi considerada a dosagem de glicose em jejum pelo método enzimático (oxidase/exoquinase). Foram considerados hiperglicêmicos os indivíduos com glicemia de jejum $\geq 126 \mathrm{mg} / \mathrm{dL}$ ou que estavam em uso de hipoglicemiantes. Em caso de alterações nos exames de sangue, o idoso era encaminhado ao posto de saúde mais próximo de sua residência para atendimento médico.

A tabulação dos dados foi feita no programa EpiData versão 3.1 (EpiData Association, Odense, Dinamarca) e a análise estatística foi realizada com auxílio do programa IBM SPSS versão 18.0 (IBM Corp., Nova York, Estados Unidos). Todas as variáveis foram qualitativas, sendo descritas em números absolutos e proporções. Para verificar associação entre as variáveis de interesse, foi aplicado o teste de quiquadrado. $\mathrm{O}$ intervalo de confiança pré-estabelecido foi de $95 \%$ e o erro $\alpha$ de $5 \%$.

O estudo foi aprovado pelo Comitê de Ética em Pesquisa da Universidade do Sul de Santa Catarina, sob registro 09.345.4.01.III, em respeito à Resolução 466/12 do Conselho Nacional de Saúde.

\section{RESULTADOS}

Foram avaliados 833 idosos, sendo $61,8 \%$ mulheres. A média de idade foi de $68,6 \pm 6,9$ anos. A prevalência de hiperglicemia foi de $26,4 \%$. A Tabela 1 apresenta os dados sociodemográficos e clínicos dos indivíduos, estratificados por glicemia de jejum $<126 \mathrm{mg} / \mathrm{dL}$ (grupo sem hiperglicemia, $\mathrm{n}=613$ ) e glicemia de jejum $\geq 126 \mathrm{mg} / \mathrm{dL}$ ou uso de hipoglicemiante (grupo hiperglicemia, $\mathrm{n}=220$ ). Dos 220 pacientes inclusos no grupo hiperglicemia, 190 estavam em uso de hipoglicemiantes orais e mesmo assim 112 apresentavam glicemia de jejum $\geq 126 \mathrm{mg} / \mathrm{dL}$; enquanto 78 apresentavam glicemia de jejum $<126 \mathrm{mg} / \mathrm{dL}$. Ainda no grupo hiperglicemia, $30 \mathrm{pa}-$ cientes apresentavam glicemia de jejum $\geq 126 \mathrm{mg} / \mathrm{dL}$ e não estavam em uso de hipoglicemiantes.

Tabela 1. Características sociodemográficas e clínicas de 833 idosos selecionados por amostragem aleatória simples entre a população de Tubarão, Santa Catarina, de setembro de 2010 a maio de 2011, e testados para hipoglicemia de jejum.

\begin{tabular}{|c|c|c|c|c|}
\hline \multirow[b]{2}{*}{ Características } & \multirow[b]{2}{*}{ Total } & \multicolumn{2}{|c|}{ Hiperglicemia* } & \multirow[b]{2}{*}{$p^{\dagger}$} \\
\hline & & $\underset{n(\%)}{\operatorname{Sim}}$ & $\begin{array}{c}\text { Não } \\
\text { n (\%) }\end{array}$ & \\
\hline \multicolumn{5}{|l|}{ Sexo } \\
\hline Masculino & 318 & $77(24,2)$ & $241(75,8)$ & \multirow{2}{*}{0,258} \\
\hline Feminino & 515 & $143(27,8)$ & $372(72,2)$ & \\
\hline \multicolumn{5}{|l|}{ Idade (anos) } \\
\hline 60-69 & 510 & $124(24,3)$ & $386(75,7)$ & \multirow{3}{*}{0,209} \\
\hline $70-79$ & 250 & $73(29,2)$ & $177(70,8)$ & \\
\hline$\geq 80$ & 73 & $23(31,5)$ & $50(68,5)$ & \\
\hline Alcoolismo & 83 & $24(28,9)$ & $59(71,1)$ & 0,168 \\
\hline Tabagismo & 335 & $90(26,9)$ & $245(73,1)$ & 0,860 \\
\hline Sedentarismo & 690 & $185(26,8)$ & $505(73,2)$ & 0,594 \\
\hline Obesidade & 299 & $103(34,4)$ & $196(65,6)$ & $<0,001$ \\
\hline Mãe diabética & 185 & $72(38,9)$ & $113(61,1)$ & $<0,001$ \\
\hline Pai diabético & 79 & $30(38,0)$ & $49(62,0)$ & $<0,001$ \\
\hline
\end{tabular}

* Glicemia de jejum $\geq 126 \mathrm{mg} / \mathrm{dL}$ ou em uso de medicamentos hipoglicemiantes. ${ }^{\dagger}$ Teste do qui quadrado.

\section{DISCUSSÃO}

O DM é uma entidade clínica que exige acompanhamento médico e educação do paciente em 
relação às comorbidades, além de integração da tríade paciente-família-profissionais de saúde para que a doença seja adequadamente controlada [16]. De acordo com a Federação Internacional de Diabetes, em 2015 o Brasil possuía 14,3 milhões de diabéticos, o que o coloca como o quarto país com o maior número de adultos com essa condição [4]. Assim, por ser um importante problema de saúde pública, o rastreio e prevenção do DM devem ser estimulados. No presente estudo, $26,4 \%$ da amostra estava em uso de hipoglicemiantes orais e/ou apresentava glicemia de jejum $\geq 126 \mathrm{mg} / \mathrm{dL}$. Apesar de isso não ser suficiente para comprovar o diagnóstico de DM, é uma frequência elevada e que merece acompanhamento dessa parcela da população.

A obesidade, definida tradicionalmente como índice de massa corpórea $\geq 30 \mathrm{~kg} / \mathrm{m}^{2}$, é uma doença metabólica crônica caracterizada pelo excesso de gordura corporal em relação à massa magra. Verificouse neste estudo associação significativa entre obesidade e hiperglicemia, visto que os obesos apresentaram mais hiperglicemia do que os não obesos. Não se pode afirmar a existência de DM nesse grupo, porém, a associação entre obesidade e DM2 já é bem demonstrada em estudos nacionais $[17,18]$ e é considerada o fator de risco modificável mais importante para o seu desenvolvimento [19].

Associam-se resistência insulínica, dislipidemia, hiperglicemia e DM2 com obesidade, principalmente obesidade abdominal. Muitas alterações metabólicas ocorrem em pacientes com obesidade e DM2; no entanto, a hiperinsulinemia e a resistência insulínica parecerem ter papel fundamental nessa relação e provavelmente também no desenvolvimento de dislipidemia e alterações nos níveis circulantes de andrógenos e estrógenos [20]. A relação entre DM2 e obesidade é complexa, mas parece estar relacionada com um pequeno grau de inflamação do tecido adiposo visceral, oriundo do aumento da secreção de adipocinas e muitas outras citocinas que propiciam a resistência insulínica, [21] e com o aumento da produção hepática de glicose pela redução da depuração da insulina [22].

O tabagismo aumenta o risco de muitas complicações do DM, incluindo cegueira, úlceras de membros inferiores, amputações e doença cardíaca. O consumo de tabaco afeta quase todos os órgãos do corpo e é a maior causa de morte evitável em todo o mundo [23]. Apesar de o tabagismo ser apontado como um dos fatores de risco para DM2, já que eleva a concentração de lipídeos no abdômen, diminui a sensibilidade à insulina e aumenta a concentração da glicemia depois de um teste oral de tolerância à glicose, este estudo não mostrou relação significativa entre hiperglicemia e tabagismo [22]. Pesquisa semelhante realizada em Viçosa (MG) também não encontrou tal associação [24].

No presente estudo, os valores de glicemia dos indivíduos que fazem atividades físicas e dos que não fazem foram semelhantes, não havendo uma relação significativa. Entretanto já se demonstrou que, por proporcionar perda de peso, a atividade física é responsável por reduzir em $30-50 \%$ o risco de desenvolver DM2. Qualquer tipo de atividade física deve ser estimulada com esse objetivo. Caminhar 150 minutos por semana, por exemplo, reduz em $60 \%$ o risco relativo para DM2 quando comparada a caminhar menos que 60 minutos durante a semana [19]. Um estudo realizado entre São Paulo, SP e Rio de Janeiro, RJ revelou que, apesar da baixa prevalência de atividade física entre diabéticos, a prática mostrou-se significativa para a prevenção do DM [25]. Além disso, a atividade física é importante para o contexto social, de saúde e de qualidade de vida do idoso, por proporcionar maior capacidade funcional e independência para atividades básicas [26].

Especialmente em fumantes e mulheres, o consumo de pequenas a moderadas quantidades de álcool está associado com um risco menor de DM2, provavelmente por aumentar a sensibilidade insulínica e reduzir a resistência a esse hormônio durante o jejum [27]. No entanto, o consumo exagerado de álcool potencializa o desenvolvimento de DM por disfunção das células beta-pancreáticas e por ser um fator de risco para cirrose hepática, que aumenta o risco para DM2 [28]. Neste trabalho, no entanto, o alcoolismo não teve relação significativa com hiperglicemia.

A presença de história familiar positiva para DM mostrou-se associada a hiperglicemia. Há uma forte ligação genética hereditária no DM2; a concordância entre gêmeos monozigóticos é aproximadamente 100\% e cerca de $25 \%$ no caso de parentes com DM2 [29]. Nosso achado é corroborado pelo trabalho de Júnior et al. [18], que mostrou relação concordante entre esses fatores. Esta variável tem importância crucial, já que é um dos fatores de risco não modificáveis para o desenvolvimento de DM.

Como limitação deste estudo, destaca-se a não realização de inquérito alimentar, já que a dieta pode influenciar na hiperglicemia. Além disso, há necessidade de repetição dos exames para determinação do diagnóstico e confirmação dos achados. Entrentanto, ressalta-se que este estudo é um rastreamento importante da situação de saúde dos idosos e que o fato de que boa parte dos participantes já 
estava em uso de hipoglicemiantes sugere um provável diagnóstico prévio de DM.

Conclui-se que uma parcela significativa dos idosos que fizeram parte do estudo foram considerados hiperglicêmicos. Entre os fatores de risco analisados, a obesidade e história familiar de DM foram significativas para a hiperglicemia. Diante disso, sugere-se a realização de estudos adicionais para analisar a prevalência de DM nessa população, a fim de favorecer o diagnóstico precoce e o tratamento adequado, prevenindo os desfechos negativos.

\section{NOTA}

Declaração de conflitos de interesse

Os autores declaram não haver conflitos de interesse relevantes ao conteúdo deste estudo.

\section{REFERÊNCIAS}

1. World Health Organization. Definition and diagnosis of diabetes mellitus and intermediate hyperglycemia: report of a WHO/IDF consultation. Geneva: WHO; 2006

2. American Diabetes Association. Classification and diagnosis of diabetes. Diabetes Care. 2016 Jan;39 Suppl 1:S13-22. http://dx.doi. org/10.2337/dc16-S005

3. Sociedade Brasileira de Diabetes. Diretrizes da Sociedade Brasileira de Diabetes: 2015-2016. São Paulo: AC Farmacêutica; 2016.

4. International Diabetes Federation. IDF Diabetes Atlas. 7ํㅡ ed. Brussels: International Diabetes Federation; 2015.

5. Ministério da Saúde (BR). Secretaria de Atenção à Saúde. Departamento de Atenção Básica. Diabetes Mellitus. Brasília: Ministério da Saúde; 2006. (Cadernos de Atenção Básica; $n^{0}$ 16. Série A - Normas e Manuais Técnicos).

6. Lind M, Wedel H, Rosengren A. Excess Mortality among Persons with Type 2 Diabetes. N Engl J Med. 2016 Fev 25;374(8):788-9. http://dx.doi.org/10.1056/NEJMc1515130

7. Evans JL, Goldfine ID. Aging and insulin resistance: just say iNOS. Diabetes. 2013 Feb;62(2):346-8. http://dx.doi.org/10.2337/db12-1239

8. Scheen AJ. Diabetes mellitus in the elderly: insulin resistance and/or impaired insulin secretion? Diabetes Metab. 2005 Dec;31 Spec No 2:5S27-5S34.

9. Kirkman MS, Briscoe VJ, Clark N, Florez H, Haas LB, Halter JB, Huang ES, Korytkowski MT, Munshi MN, Odegard PS, Pratley RE, Swift CS. Diabetes in older adults. Diabetes Care. 2012 Dec;35(12):2650-64. http://dx.doi.org/10.2337/dc12-1801

10. Chatterjee R, Narayan KM, Lipscomb J, Phillips LS. Screening adults for pre-diabetes and diabetes may be cost-saving. Diabetes Care. 2010 July;33(7):1484-90. http://dx.doi.org/10.2337/dc10-0054

11. Associação de Municípios da Região de Laguna. Municípios Associados - AMUREL [Internet]. Tubarão: 2016 [cited 2016 Sept 07]. Available from: http://www.amurel.org.br/index/municipios-associados/codMapaItem/41796

12. Instituto Brasileiro de Geografia e Estatística. IBGE - Cidades [Internet]. Tubarão; 2016 [cited 2016 Sep 07]. Available from: http://www. cidades.ibge.gov.br

13. Centers for Disease Control and Prevention. State-specific secondhand smoke exposure and current cigarette smoking among adults United States, 2008. MMWR Morb Mortal Wkly Rep. 2009 Nov 13;58(44):1232-5.

14. O'Brien CP. The CAGE questionnaire for detection of alcoholism: a remarkably useful but simple tool. JAMA. 2008 Nov 5;300(17): 2054-6. http://dx.doi.org/10.1001/jama.2008.570

15. Craig CL, Marshall AL, Sjöström M, Bauman AE, Booth ML, Ainsworth BE, Pratt M, Ekelund U, Yngve A, Sallis JF, Oja P. International physical activity questionnaire: 12-country reliability and validity. Med Sci Sports Exerc. 2003 Ago;35(8):1381-95.

16. Inzucchi SE, Bergenstal RM, Buse JB, Diamant M, Ferrannini E, Nauck M, Peters AL, Tsapas A, Wender R, Matthews DR; American Diabetes Association (ADA); European Association for the Study of Diabetes (EASD). Management of hyperglycemia in type 2 diabetes: a patient-centered approach: position statement of the American Diabetes Association (ADA) and the European Association for the Study of Diabetes (EASD). Diabetes Care. 2012 June;35(6):1364-79. http://dx.doi.org/10.2337/dc12-0413

17. Seus TLC, Siqueira FV, Silveira DS, Tomasi E, Thumé E, Silva SM, Dilelio A, Piccini RX, Facchini LA. Autorrelato de diabetes e atividade física no Brasil. Rev Bras Ativ Fis Saúde. 2012;17(6):520:31.

18. Júnior WR, Gaban SCN, Pontes ERJC, Souza CC, Gimenes LP, Lacerda PF, Cunga MLMN, Stefanello II JVL, Brum LM, Oliveira AA, Silva CR, Ribeiro ALD. Diabetes mellitus and impaired glucose tolerance in urban adult population. Rev Assoc Med Bras. 2014;60(2): 118-24. http://dx.doi.org/10.1590/1806-9282.60.02.008

19. Wu Y, Ding Y, Tanaka Y, Zhang W. Risk factors contributing to type 2 diabetes and recent advances in the treatment and prevention. Int J Med Sci. 2014 Set 6;11(11):1185-200.

20. Ferguson RD, Gallagher EJ, Scheinman EJ, Damouni R, LeRoith D. The epidemiology and molecular mechanisms linking obesity, diabetes, and cancer. Vitam Horm. 2013;93:51-98. http://dx.doi.org/10.1016/B978-0-12-416673-8.00010-1 
21. Schmidt FM, Weschenfelder J, Sander C, Minkwitz J, Thormann J, Chittka T, Mergl R, Kirkby KC, Faßhauer M, Stumvoll M, Holdt LM, Teupser D, Hegerl U, Himmerich H. Inflammatory cytokines in general and central obesity and modulating effects of physical activity. PLoS One. 2015 Mar 17;10(3):e0121971. http://dx.doi.org/10.1371/journal.pone.0121971

22. Lyra R, Oliveira M, Lins D, Cavalcanti N. Prevenção do diabetes mellitus tipo 2. Arq Bras Endocrinol Metab. 2006;50(2):239-49. http://dx.doi.org/10.1590/S0004-27302006000200010

23. Register SJ, Harrington KF, Agne AA, Cherrington AL. Effectiveness of Non-Primary Care-Based Smoking Cessation Interventions for Adults with Diabetes: A Systematic Literature Review. Curr Diab Rep. 2016 Sept;16(9):81

24. Vitoi NC, Fogal AS, Nascimento CM, Franceschini SCC, Ribeiro AQ. Prevalência e fatores associados ao diabetes em idosos no município de Viçosa, Minas Gerais. Rev Bras Epidemiol. 2015 Out;18(4):953-65.

25. Martinez MC, Latorre MRDO. Fatores de risco para hipertensão arterial e diabete melito em trabalhadores de empresa metalúrgica e siderúrgica. Arq Bras Cardiol. 2006;87(4):471-9. http://dx.doi.org/10.1590/S0066-782X2006001700012

26. Ferreira OGL, Maciel SC, Costa SMG, Silva AO, Moreira MASP. Texto Contexto Enferm. 2012 jul-set;21(3):513-8.

27. Li XH, Yu FF, Zhou YH, He J. Association between alcohol consumption and the risk of incident type 2 diabetes: a systematic review and dose-response meta-analysis. Am J Clin Nutr. 2016 Mar;103(3):818-29. http://dx.doi.org/10.3945/ajcn.115.114389

28. Kim JY, Lee DY, Lee YJ, Park KJ, Kim KH, Kim JW, Kim WH. Chronic alcohol consumption potentiates the development of diabetes through pancreatic beta-cell dysfunction. World J Biol Chem. 2015;6:1-15. http://dx.doi.org/10.4331/wjbc.v6.i1.1

29. Olokoba AB, Obateru OA, Olokoba LB. Type 2 diabetes mellitus: a review of current trends. Oman Med J. 2012 July;27(4):269-73. http://dx.doi.org/10.5001/omj.2012.68 